\title{
Discrete Model of Plasticity and Failure of Crystalline Materials
}

\author{
V. L. Busov \\ Donbass State Engineering Academy, Kramatorsk, Ukraine \\ Email: vlbusov49@gmail.com
}

How to cite this paper: Busov, V.L. (2021) Discrete Model of Plasticity and Failure of Crystalline Materials. Applied Mathematics, 12, 147-156.

https://doi.org/10.4236/am.2021.123010

Received: January 29, 2021

Accepted: March 9, 2021

Published: March 12, 2021

Copyright (c) 2021 by author(s) and Scientific Research Publishing Inc. This work is licensed under the Creative Commons Attribution International License (CC BY 4.0).

http://creativecommons.org/licenses/by/4.0/ (c) (i) Open Access

\begin{abstract}
Within the framework of a discrete model of the nuclei of linear and planar defects, the variational principles of sliding in translational and rotational plasticity, fracture by separation (cleavage) and shear (shearing) in crystalline materials are considered. The analysis of mass transfer fluxes near structural kinetic transitions of slip bands into cells, cells into fragments of deformation origin, destruction by separation and shear for fractal spaces using fractional Riemann-Liouville derivatives, local and global criteria of destruction is carried out. One of the possible schemes of the crack initiation and growth mechanism in metals is disclosed. It is shown that the discrete model of plasticity and fracture does not contradict the known dislocation models of fracture and makes it possible to abandon the kinetic concept of thermofluctuation rupture of interatomic bonds at low temperatures.
\end{abstract}

\section{Keywords}

Variational Principles of Plasticity and Destruction, Photoelectrons, Conduction Electrons, Injected Electrons, Fractal Space, Fracture Criteria

\section{Introduction}

The analysis of works [1] [2] [3] shows that during the generation of nuclei of linear and planar defects, two subsystems of electrons arise: photoelectrons knocked out of cations by an intermittent field, and intrinsic electrons of a solid. In metals, these are conduction electrons; in dielectrics and semiconductors, they are injected into the volumes of shock waves under the influence of external strong electric fields, and also arise when impurity donor atoms are introduced into the material. Here the subsystem of intrinsic electrons with thermal velocities $v_{e p}(p=d, s)$ and matrix cations is a solid-state plasma, and the subsystem of photoelectrons is a set of plane beams with velocities $\boldsymbol{V}_{\text {phe }}$, while the subsys- 
tem of pairs of photoelectrons and cations weakly coupled by Coulomb attraction, of which photoelectrons were knocked out, is a deformation plasma beams.

At large plastic deformations leading to the formation of stable fragmented structures up to critical ones, the average electron densities $n_{e p}$ and $n_{p h e}$ and the corresponding plasma frequencies $\Omega_{e p}$ and $\Omega_{p h e}$ are quantities of the same order, which leads to a fundamentally new distribution of the dielectric constant tensor $\varepsilon_{\alpha \beta}(\omega, \boldsymbol{k})$ both in space and in time. In this case, a new branch of the spectrum associated with the presence of beams is added to the main branch of the spectrum of longitudinal oscillations of the intrinsic plasma, where $\Omega_{e p}$ reflects the collective natural oscillations, and $\Omega_{\text {phe }}$-oscillations and rotations in the additional potential relief of the nuclei of linear and plane defects. Here it is necessary to note the fundamental difference in the nature of the motion of electrons in these subsystems: in their own plasma, the directions of thermal motion of electrons are equally distributed in the total solid angle, the values of their velocities in metals are not lower than $v_{F}$; and in dielectrics and semiconductors in the volumes of shock waves at electric fields near the breakdown tend to the rates of local metallization $v_{m s}, v_{m d}$. On the contrary, in the plasma of beams, the alternating (intermittent) field creates dynamic anisotropy, while the directions of the velocities $\boldsymbol{V}_{\text {phe }}$ lie in the slip planes of single crystals, and in polycrystals the appearance of a subsystem of beams is possible at threshold values of the projections of these velocities on the slip plane in individual crystallites.

Optical and electronic micro-fractography of the surface of fatigue brittle and viscous-plastic fractures of specimens from a wide range of metals and their alloys [4] [5], suggests that the geometry of such surfaces can be described by fractal functions such as Weerstrass, Takagi, and Riemann [6], while brittle fractures as a combination of terraces and steps are reduced to a superposition of saw-tooth functions or condensation of singularities, and viscous-plastic fractures to a countable number of peaks, where the left-side and right-side derivatives of the surface profile tend either to the left to $+\infty$, and to the right to $-\infty$, or vice versa.

It is also known that the macroscopic curves of tension [7] [8] and creep [9] have an intermittent jump-like shape and thus reflect the fractal nature of deformation processes of plasticity and fracture, where, at small deformations, the density of jumps $\eta_{\text {jump }}$ and the depth of load decay per jump $\Delta \sigma$ are small, and at large deformations $\eta_{\text {jump }}$ and $\Delta \sigma$ increase to 1 order.

Currently, there are several ways to describe the structural kinetic transitions "cell-fragment", "fragment-microcrack": 1) within the framework of a synergetic approach using scale invariance [10] [11], which makes it possible to relate the fractal properties of an open system far from equilibrium with deformation processes; 2) with the help of the phase transition of the crystalline state to the amorphous [12] [13], which leads to the appearance of submicrocracks in front of the top of the growing crack; 3 ) the kinetic concept of thermofluctuation rupture of interatomic bonds [14]. Here the description of the transitions is made without using the discrete model of charged particles that oscillate and rotate in potential 
valleys of the additional potential relief of the nuclei of linear and planar defects arising due to the distribution of conduction electrons [1] [2] [3].

The aim of this work is to build a physical and mathematical discrete model of structural kinetic transitions taking into account the fractality of deformation processes.

\section{Theoretical Model}

Let us consider variations in the potential relief of a crystal $V_{c}(\vec{r}, t)$ as a functional of external currents $J_{\text {osc }}^{e}, J_{\text {turn }}^{e}, J_{\text {osc }}^{\text {cat }}, J_{\text {turn }}^{\text {cat }}$ [15]. When strip, cellular, fragmented structures stable at a given level of latent energy are formed, the necessary condition for an extremum is fulfilled

$$
\delta V_{c}\left(J_{\mu}^{v}\right)=0, v=e, c a t ; \mu=o s c, t u r n
$$

On the contrary, the processes of translational, rotational plasticity and destruction are transient processes caused by the non-equilibrium of the system from the influence of external and internal electromagnetic fields when the threshold values of extraneous currents of photoelectrons (e) and cations (cat) are successively reached: $J_{\text {eosc }}^{\text {thr }}(\operatorname{trpl})$ and $J_{\text {catosc }}^{\text {thr }}($ trpl $)$-currents of oscillations and rotations with translational plasticity; $J_{\text {eturn }}^{\text {thr }}($ rotpl $)$ and $J_{\text {catturn }}^{\text {thr }}($ rotpl $)$-currents of oscillations and rotations with rotational plasticity (rotpl); $J_{v \mu}^{\text {thr }}(d s t)$-currents of oscillations and rotations during destruction. Here equality (1) turns into an inequality, and the variations $\delta J_{\mu}^{v}(\eta)$ are connected in pairs

$$
\begin{aligned}
\delta J_{\text {osc }}^{e}(\text { trpl }) & \rightleftarrows \delta J_{\text {osc }}^{\text {cat }}(\text { trpl }) \\
\delta J_{\text {turn }}^{e}(\text { trpl }) & \rightleftarrows \delta J_{\text {turn }}^{\text {cat }}(\text { trpl }) \\
\delta J_{\text {osc }}^{e}(\text { rotpl }) & \rightleftarrows \delta J_{\text {osc }}^{\text {cat }}(\text { rotpl }) \\
\delta J_{\text {turn }}^{e}(\text { rotpl }) & \rightleftarrows \delta J_{\text {turn }}^{\text {cat }}(\text { rotpl }) \\
\delta J_{\text {osc }}^{e}(d s t) & \rightleftarrows \delta J_{\text {osc }}^{\text {cat }}(d s t) \\
\delta J_{\text {turn }}^{e}(d s t) & \rightleftarrows \delta J_{\text {turn }}^{\text {cat }}(d s t)
\end{aligned}
$$

where $\eta=$ trpl;rotpl; dst and in the region of structural kinetic transitions asymptotically tend to step functions. A natural question arises: What is the physical and mathematical model of such transitions, taking into account the fractality of deformation processes? Here we assume that the deformed volume of the material is considered as a fractal space, where the equations of mass transfer with the help of electron and ion plasma waves ([3], Formulas (20), (21)) are generalized by replacing the usual differentiation operators $\partial / \partial t, \partial / \partial x_{j}$ to operators of fractional derivative (Riemann-Liouville operator) $(\partial / \partial t)^{\alpha},\left(\partial / \partial x_{j}\right)^{\alpha}$ with fractional exponent $0<\alpha<1$ ([6], p. 75). The fractional Riemann-Liouville derivatives of order $\alpha$ are left-handed

$$
\left(D_{a^{+}}^{\alpha} f\right)(x)=\frac{1}{\Gamma(1-\alpha)} \frac{\mathrm{d}}{\mathrm{d} x} \int_{a}^{x} \frac{f(t) \mathrm{d} t}{(x-t)^{\alpha}}
$$


and right-handed operators

$$
\left(D_{b^{-}}^{\alpha} f\right)(x)=-\frac{1}{\Gamma(1-\alpha)} \frac{\mathrm{d}}{\mathrm{d} x} \int_{x}^{b} \frac{f(t) \mathrm{d} t}{(t-x)^{\alpha}}
$$

where $f \equiv f_{i n j}, f_{\text {phe }}, f_{\text {cat }}$ are the distribution functions of injected electrons, photoelectrons and cations, respectively; $\Gamma(1-\alpha)-$ Gamma function. The convolution integral is written on the right-hand sides of (3) and (4); therefore, it is more convenient to consider fractional operators in $\omega, \boldsymbol{k}$-space. If we introduce the linear operator $\mathrm{d} / \mathrm{d} x$ under the sign of this integral, then its Fourier transform as $a \rightarrow-\infty$ and $b \rightarrow+\infty$ leads to the product of the Fourier components of the function $f_{\mu}^{v}$ and the power hyperbolic function $F_{g p}$ with fractional exponent. This transformation is applicable only in undeformed dielectrics and pure undoped semiconductors, where, due to the low density of free carriers, relaxation processes proceed extremely slowly. At the same time, such processes in metals are fast, spatio-temporal intervals $[a, b] \approx \tau_{r e},(5 \div 10) a_{0}$, which is caused by the equations of selective selection of frequencies $\omega_{p w}$ and wave vectors $\boldsymbol{k}_{p w}$ of plasma waves [3]

$$
\begin{aligned}
\omega_{p w}-\boldsymbol{k}_{p w} \boldsymbol{V}_{e} & =0 \\
\varepsilon_{l}\left(\vec{k}_{p w}, \omega_{p w}\right) & =0
\end{aligned}
$$

when generating linear defects. The dielectric constant near plane defects has a tensor representation $\varepsilon_{\alpha \beta}(\omega, \boldsymbol{k})$. Here, for the low-angle boundaries of inclination and rotation in the principal (normal and tangential) axes of the matrix $\varepsilon_{\alpha \beta}$, two functions $\varepsilon_{n}$ and $\varepsilon_{t}$ can be distinguished and, accordingly, two equations of selective selection. For high-angle "interfragment" boundaries of deformation origin, in particular, multi-wall Chalmers boundaries ([16], p. 456), the number of $\varepsilon_{i}$ and selection equations increases to three.

In $\omega, \boldsymbol{k}$-space near the regions of structural kinetic transitions, by analogy with [17], there is a spectrum of threshold values of frequencies $\omega_{i}$ and wave vectors $\boldsymbol{k}_{j}$, relative to which, both on the left and on the right, the dependence $F_{g p}(\omega, \boldsymbol{k})$ is completely determined by the fractional exponent $\alpha$. We represent $\alpha$ as a power series

$$
\alpha_{i}=A_{n i}\left(\frac{n_{l i}}{n_{g l i}}\right) \cdot\left[1-\left(\frac{\delta n_{v i}}{n_{v g l i}}\right)^{\beta}\right]+A_{v i}\left(\frac{V_{p h e i}}{V_{g l i}}\right) \cdot\left[1-\left(\frac{\delta V_{v i}}{V_{v g l i}}\right)^{\beta}\right], v=e, c a t
$$

where the distribution functions of photoelectrons $f_{\text {phe }}$ and cations $f_{\text {cat }}$, from which the photoelectrons were knocked out, are averaged over the local test (volumes near slip bands, boundaries of blocks, grains, fragments) volume $V_{l}$ and global (fragment, grain, crystal) volume $V_{g l}: \delta n_{v i}=\left\langle f_{v i}\right\rangle_{V_{l}}-\left\langle f_{v i}\right\rangle_{V_{g l}} \equiv n_{v l i}-n_{v g l i}$; variations in the velocities of propagation of charged particles $\delta V_{v i}=\left\langle V_{v i}\right\rangle_{V_{l}}-\left\langle V_{v i}\right\rangle_{V_{g l}} \equiv V_{v l i}-V_{v g l i}$ for the $i$-th structural kinetic transition. Here, the numerical coefficients are $0<A_{n i}, A_{V_{i}}<1$, and the power exponent $\beta$ depends primarily on the density of conduction electrons $n_{e c}$. On the other hand, $\beta$ plays the role of the Hausdorff-Besicovich fractal dimension ([6], pp. 15-56), 
therefore $\beta=1 \div 3$. Hence, in dielectrics in the absence of shock waves and strong external fields, the $\beta\left(n_{e c}\right)$ dependence is linear, in semiconductors, it is weakly nonlinear: $\beta=1 \div 2$, and in metals it goes from a quadratic to a cubic parabola: $\beta=2 \div 3$.

\subsection{Borders of Deformation Origin}

At the first stage of the theory, let us return to the well-known island model of the hereditary boundaries of a polycrystal in metals [18], where a periodic sequence of regions of good and bad conjugation of bicrystal atoms is considered. Following the adiabaticity of the model, periodic thickening and rarefaction of cations lead to corresponding variations in the density of conduction electrons. On the contrary, when boundaries of deformation origin appear according to [3], electron plasma waves are first formed according to the rules for selecting frequencies and wave vectors, to which their ionic plasma waves are shifted and stabilized. The process of redistribution of dislocations occurs in two directions: an increase in the density of such boundaries and the appearance of multi-wall boundaries, which leads to crystal fragmentation. The main feature of these boundaries is the fulfillment of the condition

$$
\left\langle f_{e c}+f_{p h e}\right\rangle_{V_{l b}} \equiv\left(n_{\text {lec }}+n_{l p h e}\right)>n_{g l} \equiv\left\langle f_{e c}\right\rangle_{V_{c}}
$$

where $V_{l b}$ and $V_{c}$ are the volumes adjacent to the interfragment boundary with the average fragment diameter $L_{f r} \approx 100 \div 200 \mathrm{~nm} \mathrm{[5]} \mathrm{[19]} \mathrm{and} \mathrm{the} \mathrm{entire}$ crystal, respectively.

Hence, the mechanism of motion of the boundary separating fragment 1 and fragment 2 is clear, if the average density of electrons in the first $n_{g l 1}<n_{g 12}$ is the same density in the second, parallel to itself towards the first fragment. Adjacent fragments form edges and junctions, at which the selective frequencies and wave vectors of plasma waves must be matched with similar values at intersecting boundaries. It should be noted that the interaction of the deformation plasma of the beams and the intrinsic plasma of a solid at large plastic deformations leads to a significant increase in the large-scale correlation energy of the relative rotation of injected electrons and photoelectrons with respect to the distribution of conduction electrons. Here, in the region of the volume of fragment boundaries, the sizes of vacancy volumes and trajectories of rotation of injected electrons and photoelectrons are of the same order [2], the moduli $\boldsymbol{V}_{p h e}, \boldsymbol{V}_{i n j}$ approach and coincide with $v_{F}$ of conduction electrons, and in critical fragmented structures they exceed it. During the transition "slip band-cell", the dislocations interact and group into rows chaotically distributed within the cell wall, where dislocations of different signs annihilate, and each of them having the same sign, according to the AFM surface profile ([3], Figure 1, two extreme sections), is central the valley and two protrusions along its edges interact in such a way that at first the overlapping protrusions of the harmonics of plasma electron waves of the nuclei of these dislocations merge into one protrusionpeak, and then their ionic plasma waves shift to this peak and stabilize it, form- 
ing a powerful linear ridge of cations substances, while the neighboring valleys deepen significantly.

At high dislocation densities $\rho=10^{10} \div 10^{11} \mathrm{~cm}^{-2}$ [5] [20], this process can occur many times. As a result, we arrive at the "cell-fragment" transition. Near this transition on the left, the density and velocity of mobile dislocations generated both upon impact and under static loading change abruptly, which means that the variations $\delta n_{i n j}, \delta n_{p h e}, \delta V_{i n j}, \delta V_{p h e}$ are jumps, and from expression (7) it follows that the exponent $\alpha \rightarrow 0$ and reaches values of $0.1-0.2$, confirmed by experiment [17]. To the right of the transition, the decisive role is played by relaxation processes, where variations $\delta n_{i n j}, \delta n_{p h e}$ change signs, and in metals, both on the left and on the right, the values of $\alpha$ are close or practically the same, in dielectrics on the right they are close to unity, and in semiconductors they have intermediate values.

\subsection{Destruction of Crystalline Materials}

The most general global criteria for the destruction of crystalline materials by detachment and shearing are

$$
\begin{gathered}
\left\langle f_{i n j}+f_{p h e}\right\rangle_{V_{l}} \equiv n_{i n j}+n_{p h e}>n_{e c} \equiv\left\langle f_{e c}\right\rangle_{V_{g l}} \\
n_{i n j}+n_{p h e}>n_{e f f} \\
V_{p h e}, V_{i n j}>v_{F}, v_{m s}, v_{m d}, \quad x_{j} \in S
\end{gathered}
$$

for a part of the surface of the interfragment boundary, the cleavage plane. Here $n_{\text {eff }}$ is the limiting density of the electronic subsystem including photoelectrons, injected electrons, conduction electrons, reflecting the small-scale correlation energy due to the Pauli principle; at interelectronic distances $r_{\text {eff }} \leq(0.1 \div 0.2) \mathrm{nm}$ [2], the potential of interelectronic repulsion $U_{e e}$ corresponds to the power function $r^{-m}(m=6 \div 9)$; in semiconductors and dielectrics, injected electrons with velocities $V_{i n j}$, first appear, and then, as the deformations grow, photoelectrons with $V_{p h e}$; also, the velocity $V_{F}$ of conduction electrons must be replaced by the velocity of injected electrons at the rates of local metallization $v_{m s}, v_{m d}$.

In dielectrics, when fractured by cleavage along the cleavage planes, criteria (10) and (11) are satisfied. Similarly, in metals, the same criteria take place during the initiation of microcracks at interfragment boundaries ([5], p. 118). Here, microcracks even in very plastic metals $(\mathrm{Al}, \mathrm{Ag})$ appear explosively-brittle. With the growth of a crack, a so-called plastic zone is formed in front of its tip ([4], p. 272), where the same sequence of deformation processes is repeated, proceeding from the beginning of the application of the load to the opening of a new section of the crack across the plastic zone. This is precisely the self-similarity of plasticity and fracture, or the scaling inherent in fractal structures. It should be noted here that the fulfillment of fracture criteria (9) - (11) in itself does not automatically mean crack opening. The opening time intervals $\Delta t_{o p}$ caused by the interelectronic repulsive potential $U_{e e}$ should be rather small compared to the time of approach of photoelectrons, injected electrons and cations from the influence of 
the potential of electrostatic attraction $U_{\text {ecat }}$ for their stabilization. In other words, the forces from $U_{e e}$ must be large enough compared to the forces from $U_{\text {ecat }}$ to prevent the incipient crack from collapsing and forming internal free surfaces with an electric double layer and surface (Tamm) states of electrons. On the other hand consider the process of forming a new section of a growing crack, which consists of two stages. At the first stage, in metals, electrons concentrated within the sharp peak of electron plasma waves $\left\langle f_{p h e}+f_{i n j}\right\rangle$ at the interfragment boundary are accelerated under the influence of $U_{e e}$ to velocities $V_{p h e}, V_{i n j} \geq v_{F}$ along the normal to this boundary. Here the conduction electrons, although they create an additional potential relief, but it does not allow to completely limit and stabilize the emerging electron fluxes near the boundary, but either slows them down to zero at distances of the order of $\Delta L_{e e} \approx 70 \div 1200 \mathrm{~nm}$ from the boundary, or they, decelerating, are reflected back from an adjacent interfragment boundary parallel to the original boundary ([5], Figure 32). At the second stage, an accumulation of cations arises near the interfragment boundary, in which the electroneutrality condition is violated, which leads to the appearance of a repulsive potential $U_{\text {catcat }}$ inside this cluster, where the corresponding ion fluxes appear, directed from the boundary into the fragment with velocities $v_{c a t} \ll V_{\text {phe }}, V_{i n j}$. As a result, an internal cavity appears, the opening $\Delta l_{c r}$ of which should be sufficient to return the hindered electron beams with subsequent stabilization of the generated ion beams for the formation of an electric double layer of the inner free surface. If the dependencies $U_{e e}\left(r_{e e}, t\right)$ and $U_{\text {catcat }}\left(r_{\text {catcat }}, t\right)$ remain similar in the process of crack opening, then the condition for the stability of the emerging crack section is maximally simplified

$$
\frac{\Delta l_{c r}}{v_{c a t}(t)} \geq \frac{\Delta L_{e e}}{V_{e}(t)}, \quad e \equiv \text { phe, inj }
$$

where $v_{\text {cat }}(t)=\frac{1}{M_{\text {cat }}} \int_{0}^{\Delta t_{o p}} \frac{\partial U_{\text {catcat }}(r, t)}{\partial r_{\text {catcat }}} \mathrm{d} t ; V_{e}(t)=\frac{1}{m_{e}} \int_{0}^{\Delta t_{o p}} \frac{\partial U_{e e}(r, t)}{\partial r_{e e}} \mathrm{~d} t ; \Delta t_{o p}$ is time interval of crack opening. Analysis (12) shows that at $\Delta l_{c r} \approx 0,7 \div 12 \mathrm{~nm}$ ([5], Figure 31), the cations should move with supersonic speeds $v_{\text {cat }} \approx 10^{4} \mathrm{~m} / \mathrm{s}$.

The fractal shape of the emerging crack surfaces is the most acceptable for its stabilization ([4], Figure 5.47 - 5.59; [5], Figure 34, Figure 35).

\section{Discussion of Results}

At first glance, the huge variety of structures and associated deformation and relaxation processes, which take place in a wide range of loads, deformations, up to destruction, seems absolutely amazing.

To date, there is no unified theory in the literature for their description with a seemingly rather simple combination of electronic and cationic subsystems for a metal bond, semiconductor atoms for a covalent bond, cations and anions of dielectrics for an ionic bond. Nevertheless, the nature of plasticity and fracture in crystalline materials still remains completely undisclosed.

In this work, only a qualitative description of deformation processes is pre- 
sented. For a quantitative consideration, it will be necessary to solve systems of equations by numerical methods.

A fundamental question arises: Is a fragment of deformation origin a quantum dot [21] [22]? First of all, we note that a quantum dot consists of a core in the form of a semiconductor (or conductor) three-dimensional microcrystal with a diameter of $d_{c p}=2-10 \mathrm{~nm}$ inside a thin shell with a significantly larger energy gap than that of its core. The energy spectrum of electrons in the nucleus of this point is discrete with an interval between neighboring levels $\Delta \varepsilon_{c p} \sim d_{c p}^{-2}$, which is characteristic of a three-dimensional potential well at $\varepsilon_{c p}<0$.

On the contrary, the distribution of injected, photo and conductivity electrons inside a fragment with $L_{f r}=100 \div 300 \mathrm{~nm}$ has a continuous spectrum, while the interfragment boundary, in contrast to the free (impenetrable) surface, is partially permeable to these electrons. The ratio of the reflection coefficients $\varkappa_{\text {ref }}$ and transmission $\varkappa_{\text {trm }}$ of electrons in the beams, and separately, depending on the misorientation angle for low-angle and high-angle boundaries of deformation origin, has yet to be found.

The scheme of the crack initiation and growth mechanism contains a number of fundamental differences:

- Based on a discrete model of charged particles;

- Reflects the interatomic potential and the type of bond between particles inherent in a given material;

- Does not contradict the well-known dislocation models of brittle fracture by Zener, Straw, Cottrell [23] and the model of dislocation replenishment by AN Orlov [24];

- Allows abandoning the kinetic concept of thermal fluctuation rupture of interatomic bonds [14], at least at low and room temperatures.

The model of plasticity and fracture presented in this work shows that the pumping energy under shock loads is redistributed as follows: the generalized space of rectangular pulses along the lines is replaced by a similar space along planes and curved surfaces, and the superposition of step functions in the form of terraces and steps is replaced by a superposition of undifferentiated peaks and ridges in the form of grooved and pit relief of fractures, at the same time, in real conditions, most often there are mixed structures from both types of relief; with the growth of cracks, exactly self-similarity of deformation processes appears. As a result, according to the destruction criteria (9) - (11), material objects are divided into separate fragments, the properties inside which are preserved. This is precisely the fractality of these objects under deformation.

\section{Conflicts of Interest}

The author declares no conflicts of interest regarding the publication of this paper.

\section{References}

[1] Busov, V.L. (2020) On Separation of Charges and Formation of Linear Structures in the Nuclei of Dislocations in Metals. Applied Mathematics, 11, 739-752. 
https://doi.org/10.4236/am.2020.118049

[2] Busov, V.L. (2020) On the Relationship of the Discrete Model of the Nuclei of Linear and Planar Defects and the Continuum Models of Defects in Crystalline Materials. Applied Mathematics, 11, 862-875. https://doi.org/10.4236/am.2020.119056

[3] Busov, V.L. and Grechkina, M.V. (2020) Plasma Model of Generation and Slip of Linear Defects in Crystalline Materials. Applied Mathematics, 11, 1167-1177. https://doi.org/10.4236/am.2020.1111079

[4] Kocanda, S. (1985) Fatigue Cracking of Metals. Wydawnictwa Naukowo Techniczne, Warszawa.

[5] Rybin, V.V. (1986) Large Plastic Deformation and Destruction of Metals. Metallurgy, Moscow.

[6] Potapov, A.A. (2005) Fractals in Radio Physics and Radar. Sampling Topology, Publishing House University Book, Moscow.

[7] Klyavin, O.V. (1974) Features of Plastic Deformation of Crystalline Bodies at Helium Temperatures. In: Startsev, V.I., Ed., Physical Processes of Plastic Deformation at Low Temperatures, the Collection of Articles, Naukova Dumka, Kyiv, 5-30.

[8] Didenko, D.A. (1974) On the Mechanism of Low-Temperature Jump-Like Deformation of Aluminum. In: Startsev, V.I., Ed., Physical Processes of Plastic Deformation at Low Temperatures, the Collection of Articles, Naukova Dumka, Kyiv, 129-138.

[9] Koval, V.A., Soldatov, V.P. and Startsev, V.I. (1974) Creep of Copper at Temperatures of 1.4-4.2 $\mathrm{K}$. In: Startsev, V.I., Ed., Physical Processes of Plastic Deformation at Low Temperatures, the Collection of Articles, Naukova Dumka, Kyiv, 339-345.

[10] Stanley, H., Conillo, A., Klein, W., et al. (1984) Critical Phenomena: Past, Present and Future. Synergetics. Mir, Moscow.

[11] Ivanova, V.S. (1989) Fracture Synergetics and Mechanical Properties. In: Ivanova, V.S., Ed., Synergetics and Fatigue Destruction of Metals, the Collection of Scientific Works, Science, Moscow, 6-29.

[12] Pavlov, V.A. (1985) Amorphization of the Structure of Metals and Alloys with an Extremely High Degree of Plastic Deformation. Physics of Metals and Metal Science, 59, 629-649.

[13] Tutnov, A.A., Dorovskiy, V.M. and Elesin, L.A. (1989) Amorphization of Crystalline Materials in the Zone in Front of the Tip of the Developing Crack. In: Ivanova V.S., Ed., Synergetics and Fatigue Destruction of Metals, the Collection of Scientific Works, Nauka, Moscow, 45-57.

[14] Regel, V.R., Slutsker, A.I. and Tamashevsky, E.E. (1974) The Kinetic Nature of the Strength of Solids. Science, Moscow.

[15] Busov, V.L. (2019) Dynamic Evolution Equations for the Cores of Linear Defects of Crystalline Materials in Colliding Solids. Physical Mesomechanics, 22, 91-96.

[16] Mirkin, L.I. (1968) Physical Foundations of Strength and Plasticity. Publishing House of Moscow State University, Moscow.

[17] Busov, V.L. and Mikheenko, D.Yu. (2015) On the Mechanism of Destruction of the Rolling Roll. Theoretical Model. Physical Mesomechanics, 18, 72-78.

[18] Orlov, A.N., Perevezentsev, V.N. and Rybin, V.V. (1980) Borders of Grains in Metals. Metallurgy, Moscow.

[19] Glezer, A.M. and Metlov, L.S. (2008) Megaplastic Deformation of Solids. Physics and Technology of High Pressure, 18, 21-35.

[20] Panin, V.E., Likhachev, V.A. and Grinyaev, Yu.V. (1985) Structural Levels of De- 
formation of Solids. Nauka Siberian Department, Novosibirsk.

[21] Yekimov, A.I. and Onushchenko, A.A. (1981) Quantum Size Effect in Three-Dimensional Microcrystals of Semiconductors. Journal of Experimental and Theoretical Physics Letters, 34, 363-366.

[22] Reed, M.A., Randall, J.N., Aggarwal, R.J., Matyi, R.J., Moore, T.M. and Wetsel, A.E. (1988) Observation of Discrete Electronic States in a Zero-Dimensional Semiconductor Nanostructure. Physical Review Letters, 60, 535-537. https://doi.org/10.1103/PhysRevLett.60.535

[23] Hirt, J. and Lotte, I. (1972) Dislocation Theory. Atomizdat, Moscow.

[24] Orlov, A.N. (1983) Introduction to the Theory of Defects in Crystals. Higher School, Moscow. 